\title{
Acceleration of diabetes development in CXC chemokine receptor 3 (CXCR3)-deficient NOD mice
}

\author{
Y. Yamada $\cdot$ Y. Okubo $\cdot$ A. Shimada $\cdot$ Y. Oikawa $\cdot$ \\ S. Yamada $\cdot$ S. Narumi $\cdot$ K. Matsushima $\cdot$ H. Itoh
}

Received: 27 September 2011 / Accepted: 5 March 2012/Published online: 11 April 2012

(C) Springer-Verlag 2012

\begin{abstract}
Aims/hypothesis The aim of this study was to understand the role of CXC chemokine receptor 3 (CXCR3), a T-helper 1(Th1) type chemokine receptor, in the pathogenesis of type 1 diabetes. Methods We observed the incidence of diabetes in $\mathrm{Cxcr} 3$ homozygous knockout mice. We compared the expression pattern of various cytokines and chemokines and the frequency of $\mathrm{FOXP}^{+}$cells in the pancreas and pancreatic lymph nodes from $\mathrm{Cxcr}^{-/-}$NOD mice and wild-type NOD mice. In addition, we observed the migration ability of $\mathrm{CXCR}^{+} \mathrm{CD}^{+}$cells to pancreatic islets upon adoptive transfer. Finally, we examined whether $\mathrm{Cxcr} 3^{+}$regulatory $\mathrm{T}$ cells (Tregs) actually suppressed the onset of diabetes in vivo. Results $\mathrm{Cxcr}^{-/-}$NOD mice developed spontaneous diabetes earlier than did wild-type NOD mice. In $\mathrm{Cxcr} 3^{-/-}$NOD mice, Tregs were more frequent in pancreatic lymph nodes and less frequent in pancreatic islets than in wild-type NOD mice. While transferred $\mathrm{CXCR} 3^{-} \mathrm{CD} 4^{+}$cells from wild-type
\end{abstract}

Y. Yamada and Y. Okubo contributed equally to this study.

Electronic supplementary material The online version of this article (doi:10.1007/s00125-012-2547-8) contains peer-reviewed but unedited supplementary material, which is available to authorised users.

Y. Yamada $\cdot$ Y. Okubo $\cdot$ A. Shimada $(\bowtie) \cdot$ Y. Oikawa

S. Yamada $\cdot$ H. Itoh

Department of Internal Medicine,

Keio University School of Medicine,

35 Shinanomachi, Shinjuku-ku,

Tokyo 160-8582, Japan

e-mail: asmd@saichu.jp

A. Shimada $\cdot$ Y. Oikawa

Department of Internal Medicine, Saiseikai Central Hospital,

Tokyo, Japan

\section{S. Narumi $\cdot$ K. Matsushima}

Department of Molecular Preventive Medicine,

University of Tokyo School of Medicine,

Tokyo, Japan
NOD mice did not infiltrate pancreatic islets of NOD-severe combined immunodeficiency (SCID) mice, $\mathrm{CXCR} 3^{+} \mathrm{CD} 4{ }^{+}$ cells from the same mice migrated into the recipient islets and contained Forkhead box P3 (FOXP3) upon adoptive transfer. Moreover, $\mathrm{CD} 4^{+} \mathrm{CD} 25^{+}$cells from wild-type NOD mice suppressed and delayed the onset of diabetes compared with those from $\mathrm{CxCr}^{-/-}$NOD mice in a cyclophosphamide-induced diabetes model system.

Conclusions/interpretation The mechanism of accelerated diabetes onset in $\mathrm{Cxcr}^{-1}$ NOD mice was considered to be due to the lack of hybrid Tregs $\left(\mathrm{CXCR} 3^{+} \mathrm{FOXP} 3^{+} \mathrm{CD} 4^{+}\right.$cells), which could effectively migrate into and regulate Th1 inflammation in local lesions under $C x c r 3$ knockout conditions.

Keywords CXCR $3 \cdot$ Hybrid Treg $\cdot$ NOD mouse $\cdot$ Regulatory T cell $\cdot$ Type 1 diabetes

\begin{tabular}{|c|c|}
\hline \multicolumn{2}{|l|}{ Abbreviations } \\
\hline CNS & Central nervous system \\
\hline CXCL9/Mig & CXC ligand $9 /$ monokine induced by IFN- $\gamma$ \\
\hline CXCL10/IP-10 & $\begin{array}{l}\text { CXC ligand } 10 / \mathrm{IFN}-\gamma \text {-inducible protein } \\
\text { of } 10 \mathrm{kDa}\end{array}$ \\
\hline CXCL11/I-TAC & $\begin{array}{l}\text { CXC ligand 11/IFN-inducible T cell } \alpha \\
\text { chemoattractant }\end{array}$ \\
\hline CXCR3 & CXC chemokine receptor 3 \\
\hline EAE & $\begin{array}{l}\text { Experimental autoimmune } \\
\text { encephalomyelitis }\end{array}$ \\
\hline FITC & Fluorescein isothiocyanate \\
\hline FOXP3 & Forkhead box P3 \\
\hline GAPDH & Glyceraldehyde 3-phosphate dehydrogenase \\
\hline $\mathrm{H} \& \mathrm{E}$ & Hematoxylin-eosin staining \\
\hline $\mathrm{mAb}$ & Monoclonal antibody \\
\hline $\mathrm{PE}$ & Phycoerythrin \\
\hline SCID & Severe combined immunodeficiency \\
\hline Th1 & T-helper 1 \\
\hline Treg & Regulatory $\mathrm{T}$ cell \\
\hline
\end{tabular}




\section{Introduction}

Type 1 diabetes is an autoimmune disease that results from $\mathrm{T}$ cell-mediated destruction of insulin-producing pancreatic beta cells [1]. It is assumed that T-helper 1 (Th1) cells play an important role in the onset of type 1 diabetes [2].

In patients with type 1 diabetes, the serum level of CXC ligand (CXCL) $10 / \mathrm{IFN}-\gamma$-inducible protein of $10 \mathrm{kDa}$ (IP10), a Th1 type chemokine, is significantly higher than in healthy controls and patients with type 2 diabetes [3]. In NOD mice, CXCL10 is not constitutively expressed on pancreatic beta cells but is upregulated in response to a typical Th1-associated cytokine, IFN- $\gamma$, as insulitis progresses [4]. Administration of CXCL10 monoclonal antibodies to a cyclophosphamide-induced NOD diabetes mouse model resulted in delayed diabetes onset [5]. Moreover, induction of anti-CXCL10 antibodies using a gene transfer system in young NOD mice also resulted in disease suppression [4]. Therefore, we speculated that the receptor for CXCL10/IP-10, CXC chemokine receptor 3 (CXCR3), must be a key protein in the pathogenesis of type 1 diabetes.

CXCR3 is a $\mathrm{G}$ protein coupled receptor with seven transmembrane domains and its gene is encoded on the $\mathrm{X}$ chromosome in both mice and humans [6,7]. Although this receptor is also expressed on $C d 8^{+} \mathrm{T}$ cells, B cells, macrophages, dendritic cells, natural killer (NK) cells and regulatory T cells (Tregs), it is expressed mainly on Th1 cells and can lead them to sites of tissue inflammation in response to CXCR3-binding chemokines, such as CXCL9/monokine induced by IFN- $\gamma$ (Mig), CXCL10/IP-10 and CXCL11/ IFN-inducible T cell $\alpha$ chemoattractant (I-TAC) [6-11]. Therefore, it is reasonable to assume that there is an amplification loop for Th1 immune responses between Th1 cells, which produce IFN- $\gamma$, and pancreatic beta cells, which produce CXCL10, in response to this cytokine, thereby attracting more $\mathrm{Cxcr} 3$-expressing Th1 cells to the affected site and amplifying the ongoing Th1 response. In fact, blockade of the CXCL10-CXCR3 interaction inhibited infiltration of lymphocytes into pancreatic islets and suppressed the onset of diabetes in a virus-induced diabetes model [12, 13]. Moreover, overexpression of CXCL10 in islets resulted in accelerated disease [14]. Thus, we speculated that knockout of CXCR3 would suppress the onset of diabetes. Surprisingly, however, instead we observed earlier diabetes onset in CXCR3deficient NOD mice, and there was clear acceleration of diabetes development, compared with wild-type NOD mice, when an adoptive transfer system was used. In this paper we discuss the importance of $\mathrm{CXCR}^{+}$Tregs, so-called 'hybrid Tregs' [15], in the regulation of type 1 diabetes, providing a possible explanation for the observations discussed above.

\section{Methods}

Animals We purchased NOD and NOD-SCID mice from CLEA Japan (Tokyo, Japan). A female C57Bl/6 background CXCR3-deficient mouse was generated as previously described [16]. The mice were backcrossed to male NOD mice, and $\mathrm{F} 1$ female mice were again backcrossed to male NOD mice. From the F2 generation, heterozygous female mice were chosen and then backcrossed to male NOD mice. This manipulation was repeated until the sixth generation. At the fifth generation (F5), mice were screened for polymorphic microsatellite markers to determine whether they had the NOD gene background and we confirmed that they had NOD type on all markers shown in the electronic supplementary material (ESM) Fig. 1 [17]. Cxcr3 homozygous knockout female NOD mice were generated by crossing F6 knockout male and heterozygous female mice (speed congenic method). We housed all mice under specific pathogen-free conditions. All studies were approved by the Animal Care and Use Committee of Keio University.

Genotyping DNA was extracted from a small portion of the tail of experimental mice. A QIAGEN DNeasy kit (Qiagen, Courtaboeuf, France) was used for DNA isolation, followed by PCR using TaKaRa Ex Taq (TAKARA BIO, Shiga, Japan). Genotyping was performed using primers recognising $\mathrm{Cxcr} 3$ genomic DNA sequences or inserted genomic DNA sequences named $\mathrm{Neu}$. The primers shown in ESM Table 1 were used. The wild-type allele yielded a 589 bp DNA fragment and the knockout allele a $320 \mathrm{bp}$ fragment, which were analysed using agarose gels and detected by ethidium bromide (EtBr) staining. Flow cytometric analysis confirmed that CXCR3 on splenocytes was decreased in heterozygous mice and was undetectable in knockout mice (ESM Fig. 2).

Definition of diabetes All animals were assessed for glycosuria at least once a week in the evening, and plasma glucose was measured when glycosuria was observed. Being positive for glycosuria and having a plasma glucose level higher than $13.9 \mathrm{mmol} / \mathrm{l}$ confirmed diabetes.

Histological examination The pancreas was removed from mice, fixed in $10 \%$ formaldehyde and embedded in paraffin. Thin sections at four levels, $200 \mu \mathrm{m}$ apart, were cut for hematoxylin-eosin (H\&E) staining to evaluate the degree of cell infiltration in the islets by light microscopy. The pancreatic islets were scored using the following rankings: grade 0 , normal; grade 1, peri-insulitis (mononuclear cells surrounding islets and ducts, but no infiltration of the islet architecture); grade 2, moderate insulitis (mononuclear cells infiltrating $<50 \%$ of the islet architecture); and grade 3 , severe insulitis (mononuclear cells infiltrating $>50 \%$ of the islet architecture). 
Quantitative RT-PCR Total RNA was extracted from the pancreas, pancreatic lymph nodes (pLN) and spleen using an RNeasy Mini kit (Qiagen). During the procedure, DNase treatment was performed according to the manufacturer's protocol. The extracted RNA was reverse transcribed using Not I-d(T)18 primer and a First-Strand cDNA synthesis kit (GE Healthcare, Little Chalfont, UK), according to the manufacturer's instructions. Quantitative RT-PCR was conducted for Il4, Il10, Tgf- $\beta$ (also known as Tgfbl), Foxp3, Ifn- $\gamma$ (also known as Ifng), Il2, Gzmb, Ccr5 and Gapdh (internal control) in an ABI Prism 7700 sequence detector (Applied Biosystems, Foster City, CA, USA). The primers were purchased from Applied Biosystems. All reactions were performed using TaqMan Universal MasterMix (Applied Biosystems). The expression of each gene was normalised to that of Gapdh.

Immunohistochemical study The pancreases removed from NOD mice were inflated with optimal cutting temperature (OCT) compound and snap-frozen in liquid nitrogen. Following this, 4\% paraformaldehyde (PFA)-fixed $6 \mu \mathrm{m}$ thick fresh frozen tissue sections were blocked with $5 \%$ goat serum diluted in PBS. The sections were incubated with polyclonal guinea pig anti-swine insulin antibody (DakoCytomation, Kyoto, Japan), fluorescein isothiocyanate (FITC)-labelled rat anti-mouse CD4 monoclonal antibody (mAb) (H129.19; BD Pharmingen, San Diego, CA, USA), rat anti-mouse FOXP3 antibody (MF333F; Alexis Biochemicals, Plymouth Meeting, PA, USA), rabbit antimouse FOXP3 antibody (PA1-46126; Thermo Fisher Scientific, Rockford, IL, USA), and phycoerythrin (PE)-labelled rat anti-mouse CXCR3 mAb (R\&D Systems, Minneapolis, MN, USA), followed by Alexa Fluor-594-labelled goat antiguinea pig IgG, Alexa Fluor-488-labelled goat anti-rat IgG and Alexa Fluor-568-labelled goat anti-rabbit IgG (Molecular Probes, Eugene, OR, USA). The sections were then examined by fluorescence microscopy. We ensured staining specificity by using control slides in which incubation with the primary antibody was skipped. Thin sections at three levels, $200 \mu \mathrm{m}$ apart, were cut to evaluate the islets.

Flow cytometry Cell surfaces were stained with the following directly conjugated antibodies specific for mouse proteins: FITC or PE-labelled anti-mouse CD4 mAb (H129.19; BD Pharmingen) or PE-labelled anti-mouse CXCR3 mAb (R\&D Systems). Mouse FOXP3 staining was performed with a FITC anti-mouse FOXP3 staining set (eBioscience, San Diego, CA, USA).

To perform flow cytometric analyses of pancreases, approximately $2.5 \mathrm{ml}$ of cold HBSS (Sigma-Aldrich, St Louis, MO, USA) containing collagenase XI $(1 \mathrm{mg} / \mathrm{ml}$; SigmaAldrich) was injected into the common bile duct. The distended pancreas was removed and incubated at $37^{\circ} \mathrm{C}$ for
17 min with shaking. Cold HBSS was added to stop the digestion. The pancreas was then dissected using a cell strainer and stained with the same monoclonal antibodies as the spleen cell preparations. The stained cells were analysed with an Epics XL-MCL (Beckman Coulter, Brea, CA, USA).

Suppression assay We evaluated the suppressive capacity of Tregs $\left(\mathrm{CD} 4^{+} \mathrm{CD} 25^{+}\right.$cells) against proliferation of effector cells $\left(\mathrm{CD} 4^{+} \mathrm{CD} 25^{-}\right.$cells) using a carboxyfluorescein diacetate succinimidyl ester (CFSE) labelling system in vitro [18]. We conducted this experiment using splenocytes and lymphocytes from pancreatic lymph nodes each separately. $\mathrm{CD} 4^{+} \mathrm{CD} 25^{+}$and $\mathrm{CD} 4^{+} \mathrm{CD} 25^{-}$cells were purified using a MACS system (Miltenyi Biotec, Bergisch Gladbach, Germany). We stimulated $\mathrm{CD} 4^{+} \mathrm{CD} 25^{-}$cells (spleen: $3.5 \times$ $10^{5}$ cells, pancreatic lymph nodes: $1.3 \times 10^{4}$ cells) from 12 week-old wild-type female NOD mice with a Dynabeads mouse CD3/CD28 T cell Expander (Invitrogen, Carlsbad, CA, USA) and simultaneously added Tregs $\left(\mathrm{CD} 4^{+} \mathrm{CD} 25^{+}\right.$ cells; spleen: $>3.5 \times 10^{4}$ cells, pancreatic lymph nodes: $>6.5 \times 10^{3}$ cells) from wild-type female NOD or $\mathrm{Cxcr}^{-1}$ female NOD mice. These cells were cultured for $48 \mathrm{~h}$ in RPMI 1640 medium supplemented with $10 \%$ heatinactivated FBS and penicillin/streptomycin. These cells were then analysed with an Epics XL-MCL (Beckman Coulter).

Cell transfer For the adoptive transfer experiments, the spleens of non-diabetic and diabetic $\mathrm{Cxcr}^{-/-}$and wildtype 14-18-week-old NOD mice were removed. After lysis of erythrocytes, the splenocytes were washed with PBS. The splenocytes were then resuspended at an appropriate concentration for transfer $\left(8.0 \times 10^{6} \mathrm{cells} / \mathrm{ml}\right)$, and $250 \mu \mathrm{l}$ of the cell suspension (including $2.0 \times 10^{6}$ cells) was injected intraperitoneally into each NOD-SCID recipient.

For the migration study, the spleens of 27-week-old diabetic wild-type NOD mice were removed. After lysis of erythrocytes, the splenocytes were washed with PBS and incubated with FITC-labelled anti-mouse CD4 mAb (H129.19; BD Pharmingen) and PE-labelled anti-mouse CXCR3 $\mathrm{mAb}$ (R\&D Systems). CXCR $3^{-} \mathrm{CD} 4^{+}$and CXCR $3^{+} \mathrm{CD} 4^{+}$cells were isolated by sorting using an Altra HyperSort System (Beckman Coulter). The splenocytes were then resuspended at an appropriate concentration for transfer $\left(8.0 \times 10^{5}\right.$ cells $\left./ \mathrm{ml}\right)$, and $250 \mu \mathrm{l}$ of the cell suspension (including $2.0 \times 10^{5}$ cells) was injected intraperitoneally into each NOD-SCID recipient. Eleven weeks later, the recipients were killed and the pancreas was examined histologically.

For regulatory $\mathrm{T}$ cell transfer study, we used MACS (Miltenyi Biotec) sorted $\mathrm{CD} 4^{+} \mathrm{CD} 25^{+}$cells as regulatory $\mathrm{T}$ cells. Nine to 12-week-old female non-diabetic NOD mice 
were injected intraperitoneally with cyclophosphamide (Shionogi, Osaka, Japan, $225 \mathrm{mg} / \mathrm{kg}$ body weight) on day 0 and injected intraperitoneally with $\mathrm{CD} 4^{+} \mathrm{CD} 25^{+}$cells (0.6-1.0 $\times 10^{6}$ cells/mouse) of $\mathrm{CxCr}^{-/}$or wild-type 12-14week-old female NOD mice on day 3. All animals were assessed for glycosuria daily in the evening, and plasma glucose was examined when glycosuria was observed. Diabetes was confirmed when glycosuria was positive and plasma glucose level was higher than $13.9 \mathrm{mmol} / \mathrm{l}$.

Statistical analysis Results are presented as mean \pm SEM. The $\chi^{2}$ test or Fisher's exact probability test were used to compare the incidence of diabetes. The $\chi^{2}$ test was used to compare the frequency of $\mathrm{FOXP}^{+}$cells among $\mathrm{CD} 4^{+}$cells in and around the islets. Other mean values in the groups of $\mathrm{CxCr}^{-/-}$NOD mice and wild-type NOD mice were compared using the Mann-Whitney $U$ test. A $p$ value less than 0.05 was considered to indicate a statistically significant difference.

\section{Results}

Earlier onset of diabetes in $\mathrm{Cxcr}^{-/-}$NOD mice First, we compared the incidence of diabetes between $\mathrm{Cxcr3}^{-/}$NOD and wild-type NOD mice. As shown in Fig. 1, the onset of diabetes was significantly earlier in $\mathrm{Cxcr}^{-/-}$NOD than in wild-type NOD mice (55\% vs $24 \%$ at 28 weeks of age, $p=$ 0.04). Moreover, histological examination of the pancreas revealed a higher degree of insulitis in 12-week-old $\mathrm{Cxcr}^{-/}$ NOD mice than in age-matched wild-type NOD mice (pancreatic islet number with score grade 3/total pancreatic islet number: $\mathrm{Cxcr3}^{-/-} 27.3 \%$ (38/139) vs wild-type $8.9 \%$ (16/180), $p<0.0001$, ESM Fig. 3). Furthermore, when we transferred $\mathrm{Cxcr}^{-/-}$NOD splenocytes into NOD-SCID mice, the incidence of diabetes was higher $\left(\mathrm{CxCr}^{-/-}\right.$non-

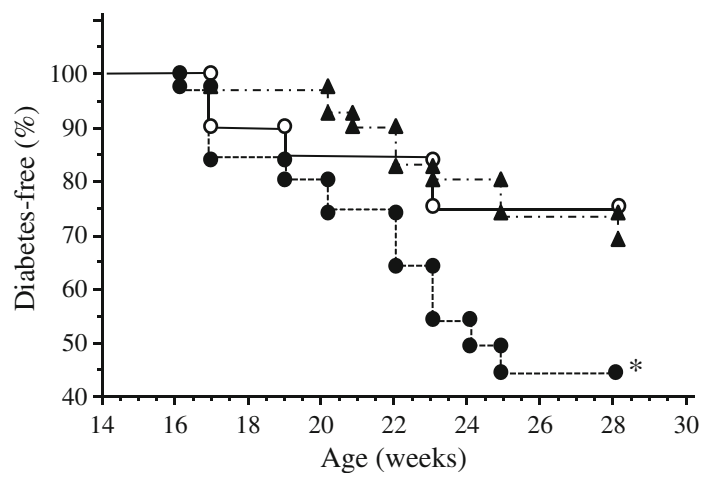

Fig. $1 \mathrm{Cxcr}^{-/-}$NOD mice developed diabetes earlier than $\mathrm{CxCr}^{+/-}$or wild-type NOD mice. Kaplan-Meier plot of cumulative diabetes incidence in female wild-type (white circles, $n=21$ ), $C x c r 3^{+/-}$ (black triangles, $n=31$ ) and $\mathrm{Cxcr}^{-1-}$ NOD mice (black circles, $n=20$ ). ${ }^{*} p<0.05$ vs wild-type NOD group at 28 weeks of age, by $\chi^{2}$ test diabetic $100 \%$ vs wild-type non-diabetic $10 \%$ at 9 weeks after transfer, $p<0.01, \mathrm{Cxcr}^{-/-}$diabetic $100 \%$ vs wild-type diabetic $42.1 \%$ at 9 weeks after transfer, $p=0.01$, Fig. 2) and the onset was earlier than in NOD-SCID recipients of wildtype NOD splenocytes, confirming that $\mathrm{Cxcr}^{-/-}$NOD spleen cells were clearly more immunologically active than wild-type NOD spleen cells.

Mirror image expressions of Treg element in pancreatic lymph nodes and pancreas To gain insight into the earlier onset of diabetes in $\mathrm{Cxcr}^{-/}$NOD mice, we examined cytokine expression levels in the pancreatic lymph nodes and pancreas from prediabetic 12-week-old $\mathrm{Cxcr}^{-/}$NOD mice, which are considered to represent the stage immediately before the onset of diabetes, and compared the findings with those in age-matched wild-type NOD mice. As shown in Fig. 3, expression levels of Tgf- $\beta$, Foxp3, Ill0 and Il4 were higher in pancreatic lymph nodes from $\mathrm{Cxcr}^{-/-}$NOD mice, whereas expression levels were lower in the pancreas, with these levels being a 'mirror image' of each other. Moreover, immunohistochemical examination of pancreatic islets revealed that the frequency of $\mathrm{FOXP}^{+}$cells among $\mathrm{CD}^{+}$cells, which surrounded the pancreatic islets, was higher in wild-type than in $\mathrm{Cxcr}^{-/-}$islets $\left(\mathrm{FOXP}^{+} \mathrm{CD}^{+}\right.$ cells/ CD4 ${ }^{+}$cells: $\mathrm{Cxcr}^{-/-} 13.7 \pm 1.7 \%$ vs wild-type $23.5 \pm$ $2.8 \%, p<0.05$, Figs 4 and 5 ). In addition, the frequency of $\mathrm{FOXP}^{+}$cells among $\mathrm{CD}^{+}$cells was higher in pancreatic lymph nodes from $\mathrm{Cxcr}^{-/-}$NOD mice, as demonstrated by flow cytometry $\left(\mathrm{Cxcr} 3^{-/-} 10.01 \pm 0.60 \%\right.$ vs wild-type $8.51 \pm$ $0.43 \%, p<0.05, n=9$ /group, ESM Fig. 4). These results suggest that CXCR3 is required for effective migration of $\mathrm{FOXP}^{+}$cells into the pancreas itself.

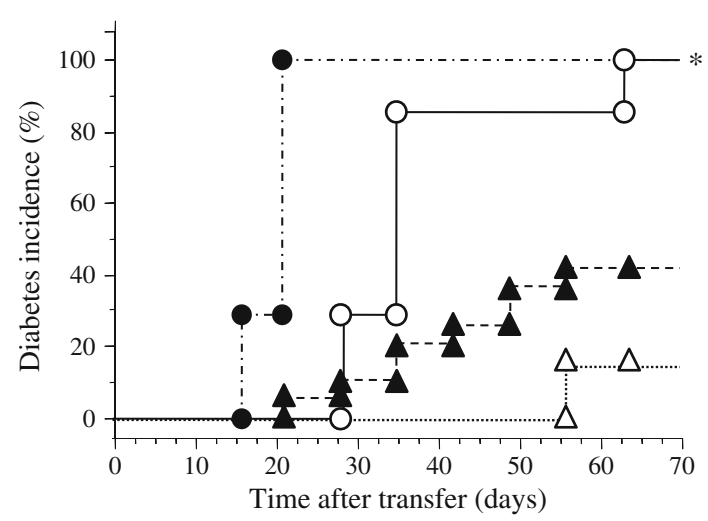

Fig. 2 Splenocytes from $\mathrm{Cxcr}^{-1-}$ NOD mice are more diabetogenic than those from wild-type NOD mice. Kaplan-Meier plot of cumulative diabetes incidence in female NOD-SCID mice after adoptive transfer. White circles: $\mathrm{Cxcr}^{-/}$non-diabetic donors $(n=7)$; black circles: $\mathrm{CxCr}^{-/-}$diabetic donors $(n=7)$; white triangles: wild-type non-diabetic donors $(n=10)$; black triangles: wild-type diabetic donors $(n=19){ }^{*} p<0.01$ vs wild-type non-diabetic group and $p=0.01$ vs wildtype diabetic group at 9 weeks after adoptive transfer, by Fisher's exact probability test 
Fig. 3 In $\mathrm{Cxcr}^{-/-}$NOD mice $(\mathrm{KO})$, regulatory $\mathrm{T}$ cells accumulate in pancreatic lymph nodes (pLN) but not in pancreas (Panc), compared with wild-type (WT) NOD mice. Expression of Treg-associated cytokines and chemokines in $\mathrm{pLN}$ and pancreas in 12-week-old $\mathrm{Cxcr}^{-/-}$mice (white bars) and WT NOD mice (black bars) ( $n=4 /$ group). a Tgf- $\beta$, (b) Foxp3, (c) Il10, (d) Il4. Data are shown as mean \pm SEM. ${ }^{*} p<0.05$ by Mann-Whitney $U$ test a

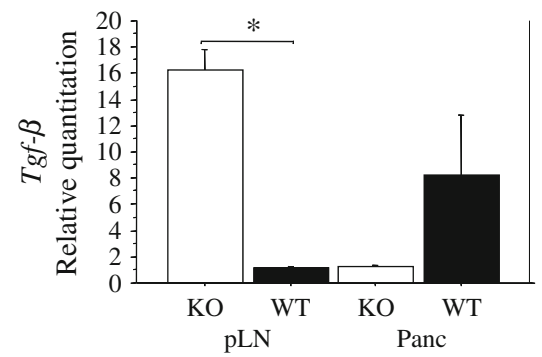

C

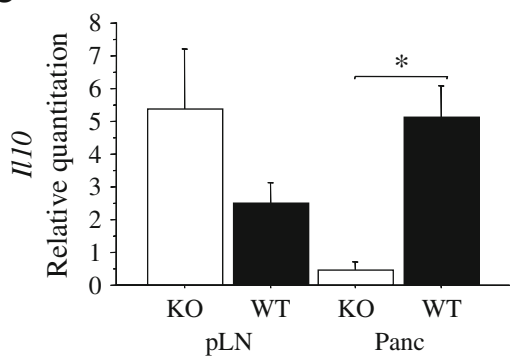

b

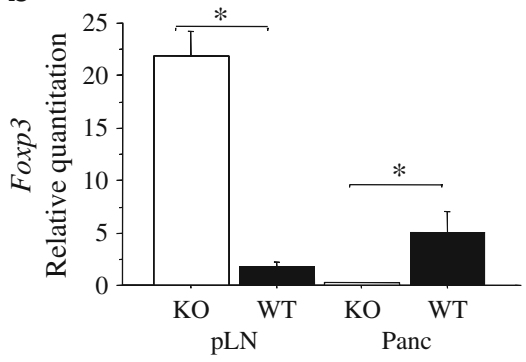

d

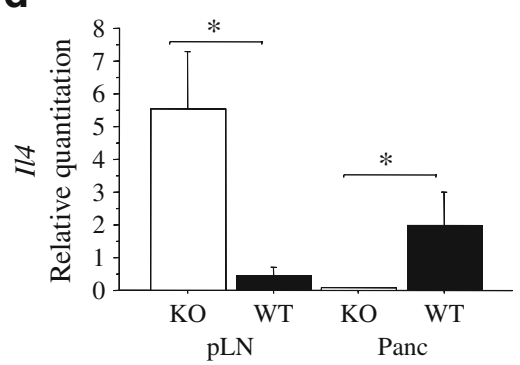

$\mathrm{CXCR}^{+} \mathrm{CD}^{+}$cells migrate into recipient islets with adoptive transfer First of all, we confirmed the presence of $\mathrm{CXCR}^{+} \mathrm{FOXP}^{+}$cells in prediabetic (20 weeks old) wildtype NOD islets (ESM Fig. 5). We transferred CXCR3 ${ }^{+} \mathrm{CD}^{+}$ cells from 27-week-old diabetic wild-type NOD mice into NOD-SCID recipients, and found that $\mathrm{CXCR} 3^{+} \mathrm{CD} 4^{+}$cells migrated into the recipient islets (Fig. 6a). These infiltrating cells were confirmed to be $\mathrm{CD}^{+}$cells (Fig. 6c). Furthermore, a portion of these cells contained FOXP3 (Fig. 6d). Thus, $\mathrm{CXCR}^{+}{ }^{\mathrm{FOXP}} 3{ }^{+} \mathrm{CD} 4{ }^{+}$cells were confirmed to migrate into recipient islets. On the other hand, when we transferred $\mathrm{CXCR}^{-} \mathrm{CD}^{+}$cells from the same donor into NOD-SCID recipients, there was no cell infiltration in the recipient islets by 11 weeks post-transfer (Fig. 6b), indicating that CXCR3 is required for $\mathrm{CD}^{+}$cell migration into islets (pancreatic islet number in which insulitis occurred/total pancreatic islet number: $\mathrm{CXCR}^{+}$cells: 22/100 vs CXCR3 ${ }^{-}$cells: 0/94, $p<0.0001$ ).
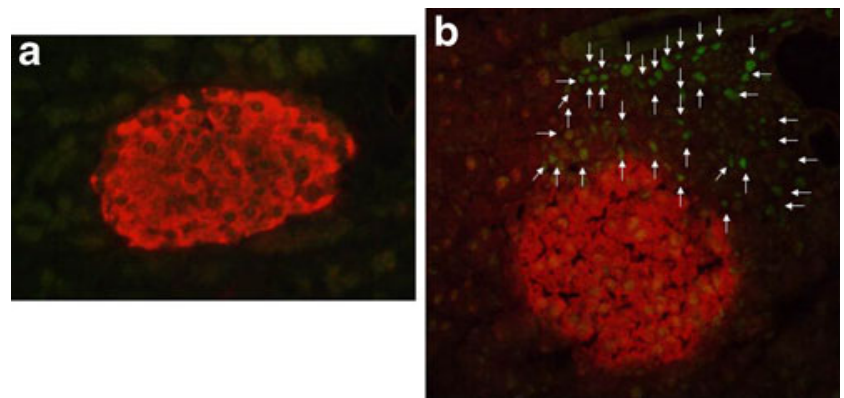

Fig. $4 \mathrm{FOXP}^{+}$cells are rare in pancreatic islets from $\mathrm{Cxcr}^{-1-} \mathrm{NOD}$ mice (a) but are present in those from wild-type NOD mice (b). FOXP3-positive cells (green, shown by arrows) infiltrating around pancreatic islets (red) were evaluated at 12 weeks of age (magnification, $\times 200)$ in both groups $(n=5 /$ group $)$. Representative islets are shown
Suppressive function of Tregs is similar in $\mathrm{Cxcr}^{-1-} \mathrm{NOD}$ mice and $\mathrm{Cxcr}^{+/+}$NOD mice In order to evaluate the difference in Treg function between $\mathrm{Cxcr}^{-/-}$NOD mice and wild-type NOD mice, we used $\mathrm{CD} 4^{+} \mathrm{CD} 25^{+}$cells from spleen or pancreatic lymph nodes as Tregs. These cells were cultured with purified $\mathrm{CD} 4^{+} \mathrm{CD} 25^{-}$cells (effector cells) from a wild-type NOD mouse to evaluate their suppressive capacity against the proliferation of the effector cells. We found no significant difference in the suppressive capacity of Tregs from either spleen or pancreatic lymph nodes between $\mathrm{CxCr}^{-/-}$and wild-type NOD mice; the suppression rate for proliferation of effector cells by Tregs, which were added at a ratio of one tenth or more of effector cells, was as follows: spleen; $C x c r 3^{-/} 23.4 \pm 3.2 \%(n=4)$, wildtype $27.9 \pm 2.4 \%(n=4)$, pancreatic lymph nodes; $C x c r 3^{-/}$ $63.0 \%$, wild-type $51.9 \%$. a

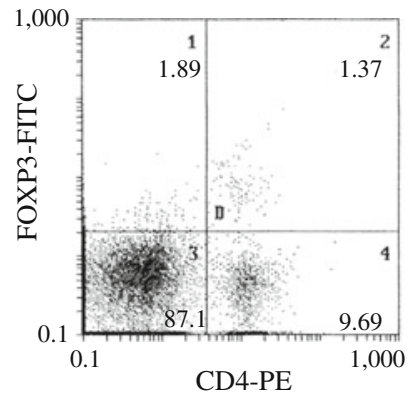

b

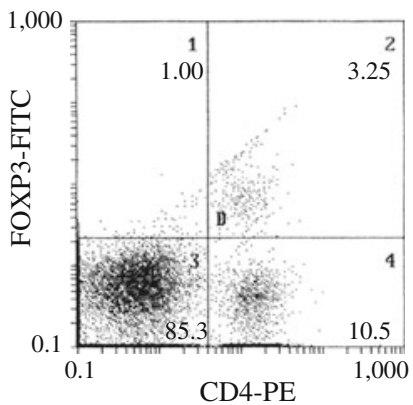

Fig. 5 Comparison of $\mathrm{FOXP}^{+} \mathrm{CD}^{+}$cell staining of $\mathrm{CxCr}^{-/-}$ NOD pancreas (a) and wild-type NOD pancreas (b). Representative flow cytometric data are shown. $X$-axis: CD4-PE, $Y$-axis: FOXP3-FITC. Numbers in quadrants indicate the percentage of cells in each 


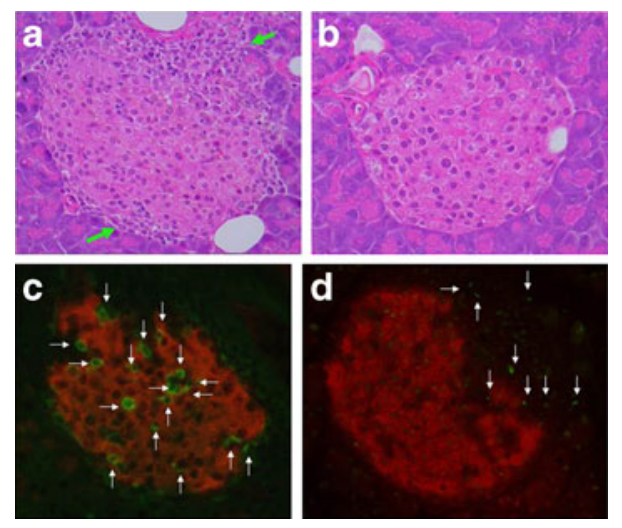

Fig. $6 \mathrm{CXCR} 3{ }^{+} \mathrm{FOXP} 3{ }^{+} \mathrm{CD} 4^{+}$cells migrate into recipient islets with adoptive transfer. $\mathrm{CD}^{+} \mathrm{CXCR}^{+}$cells (positive group) or $\mathrm{CD}^{+}{ }^{+} \mathrm{CXCR}^{-}{ }^{-}$cells (negative group) from 27 -week-old diabetic NOD mice were injected intraperitoneally into NOD-SCID recipients. a,b $H \& E$ staining of pancreatic islets of positive group (a) and negative group (b) (magnification, $\times 400$ ). Arrows indicate infiltrating lymphocytes. c Immunohistochemical staining in positive group (magnification, $\times 200$ ). $\mathrm{CD}^{+}$cells (green, arrows) have infiltrated pancreatic islets (red). d Immunohistochemical staining in positive group (magnification, $\times 200$ ). Infiltration of $\mathrm{FOXP}^{+}$cells (green, arrows) are observed around pancreatic islets (red)

Regulatory $T$ cells from $\mathrm{Cxcr}^{+/+}$NOD mice suppress and delay the onset of diabetes compared with those from $\mathrm{Cxcr}^{-1-}$ NOD mice To evaluate whether CXCR $3^{+}$Tregs actually suppress the onset of diabetes in vivo, we transferred $\mathrm{CD} 4^{+} \mathrm{CD} 25^{+}$cells $\left(0.6-1.0 \times 10^{6}\right.$ cells $)$ from wild-type mice into cyclophosphamide $(225 \mathrm{mg} / \mathrm{kg} /$ mouse) injected 9-12-week-old female NOD mice 3 days post cyclophosphamide injection and compared the response to that with transfer of a matched number of Tregs from $\mathrm{Cxcr}^{-/-} \mathrm{NOD}$ mice. As a result, we observed significantly delayed onset of diabetes in wild-type Treg-transferred mice ( $13.0 \pm 0.6$ days)

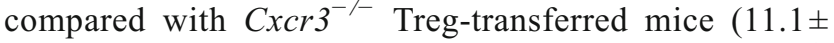
0.5 days, $p<0.05$, ESM Fig. 6). Moreover, 6 of $20 \mathrm{Cxcr}^{-/}$ Treg-transferred mice developed diabetes by 11 days post cyclophosphamide injection, whereas none of the wild-type Treg-transferred mice developed diabetes at the same time point $(p=0.02)$. The incidence of diabetes at 18 days post cyclophosphamide treatment in NOD mice without transfer was $42 \%(8 / 19)$ in this system. These results indicate that the presence of CXCR3 in Tregs effectively suppresses diabetes in vivo.

\section{Discussion}

Numerous reports $[2,19-22]$ have clarified that Th1 cells are intimately involved in the onset of type 1 diabetes. In this study, we generated NOD mice lacking CXCR3, which has been considered to make a major contribution to the cytotoxic functions of Th1 cells, and we anticipated that diabetes onset in these mice would be delayed or suppressed.
The results, however, were entirely the opposite of our expectations.

Other researchers have investigated the role of CXCR3 by using $\mathrm{Cxcr} 3$ gene deficient mice. Gao et al. [23] reported that, in a bleomycin-induced lung injury model, $\mathrm{Cxcr} 3$ knockout mice were protected from lung injury as evidenced by lower accumulation of inflammatory cells in the airways and lung interstitium than in their wild-type littermates. As with our initial expectation, they also assumed that CXCR3 signalling would promote inflammatory cell recruitment and initiate an inflammatory cytokine cascade following endotracheal bleomycin administration. In this study we examined the mRNA expression level of a Th1-type cytokine, Ifn- $\gamma$, in the pancreases from $\mathrm{Cxcr}^{--}$mice, and found the level to be similar to that in wild-type mice (data not shown). This result suggested that CXCR3deficient Th1 cells migrated in the same manner as wildtype Th1 cells, using other chemokine receptors independent of CXCR3, such as CCR5, which we confirmed to be present in pancreases from $\mathrm{Cxcr}^{-/-}$NOD mice (data not shown).

On the other hand, Müller et al. [11] reported that, in the central nervous system (CNS) in experimental autoimmune encephalomyelitis (EAE), the times to onset and peak disease severity were similar in $\mathrm{Cxcr} 3$ knockout and wild-type animals. However, $\mathrm{Cxcr} 3$ knockout mice had more severe chronic disease, with increased demyelination and axonal damage. Although the numbers of $C D 4^{+}$and $C D 8^{+} \mathrm{T}$ cells infiltrating the CNS were similar in $\mathrm{Cxcr} 3$ knockout and wild-type mice, $\mathrm{FOXP}^{+}$regulatory T cells were significantly reduced in number and were more dispersed in $\mathrm{Cxcr} 3$ knockout mice. The authors concluded that, in EAE, CXCR3 signalling kept $\mathrm{T}$ cells within the perivascular space in the CNS and augmented regulatory $\mathrm{T}$ cell recruitment and interaction between regulatory $\mathrm{T}$ cells and effector $\mathrm{T}$ cells, thereby limiting autoimmune-mediated tissue damage. In our experimental system, mRNA expression patterns clarified the marked differences in location of regulatory $\mathrm{T}$ cells between $\mathrm{Cxcr} 3^{-/-}$and wild-type NOD mice. In $\mathrm{Cxcr} 3^{-/-}$mice, regulatory $\mathrm{T}$ cells accumulated in pancreatic lymph nodes, with fewer being detected in pancreatic islets. These findings suggest that CXCR3 is a chemokine receptor for regulatory $\mathrm{T}$ cell migration into pancreatic islets, and that the earlier onset of diabetes in $\mathrm{CxCr}^{-/}$mice may be due to lack of regulatory $\mathrm{T}$ cells in pancreatic islets.

Very recently, CXCR $3^{+}$Tregs, so-called 'hybrid Tregs' [15], were reported to efficiently downregulate the Th1 inflammatory state. This concept is entirely consistent with our observations. According to the report on hybrid Tregs, $\mathrm{CXCR}^{+}{ }^{+} \mathrm{FOXP} 3^{+} \mathrm{CD} 4^{+}$cells were generated from FOXP $3^{+} \mathrm{CD} 4^{+}$cells in response to strong Th1 inflammation, particularly IFN $-\gamma$. These new-born $\mathrm{CXCR} 3{ }^{+} \mathrm{FOXP} 3^{+} \mathrm{CD} 4^{+}$ 
cells (hybrid Tregs) are unique cells, having both the migratory capacity of Th1 cells and the regulatory function of regulatory $\mathrm{T}$ cells, and these cells migrate into Th1inflammatory sites depending on the Th1-chemokine gradient, as do Th1 cells, and effectively suppress the function of Th1 cells as regulatory T cells. It is efficient that naive Tregs differentiate in response to an inflammatory environment and express the chemokine receptor of their target cells. In our system, CXCR3-deficient regulatory $\mathrm{T}$ cells did not have the capacity to migrate into Th1-inflammatory sites (pancreatic islets), and accumulated in pancreatic lymph nodes and could not effectively suppress Th1 activity.

As described above, when we transferred $\mathrm{CXCR}^{+} \mathrm{CD}^{+}$ cells from 27-week-old diabetic NOD mice, considered to be in the so-called 'malignant phase' [24] (in other words an active diabetic phase) into NOD-SCID recipients, FOXP $3^{+} \mathrm{CD}^{+}$cells were observed in the recipient islets. On the other hand, when we transferred the same phenotypic population, $\mathrm{CXCR}^{+} \mathrm{CD}^{+}$cells from 10 -week-old NOD mice, considered to be in a 'benign phase' [24] (in other words a less active non-diabetic phase) no $\mathrm{FOXP}^{+} \mathrm{CD}^{+}$ cells were observed in islets, although $\mathrm{CD} 4^{+}$cells infiltrated pancreatic islets (data not shown). Moreover, the frequency of $\mathrm{CXCR}^{+}{ }^{+} \mathrm{FOXP} 3^{+}$cells was significantly higher in both pancreatic lymph nodes and among splenocytes from 29week-old (malignant phase) NOD mice than in those from 10-week-old (benign phase) NOD mice (ESM Fig. 7). Taken together, these results suggest that $\mathrm{CXCR} 3^{+} \mathrm{FOXP} 3^{+} \mathrm{CD}^{+}$ cells are generated mainly in the malignant phase, in response to IFN- $\gamma$. As we reported previously, the IFN- $\gamma$ response increased immediately prior to (or after) the onset of diabetes [2]. In general, the IFN- $\gamma$ response in NOD mice during the non-diabetic phase is lower than that in the normal strain, whereas the IFN- $\gamma$ response recovers to a 'normal' level at approximately the time of diabetes onset [2]. Our previous observations support our current hypothesis that $\mathrm{CXCR}^{+}{ }^{+} \mathrm{FOXP} 3^{+} \mathrm{CD} 4{ }^{+}$cell generation coincides with or occurs shortly after the onset of diabetes.

As mentioned in the Introduction, blockade of the CXCL10-CXCR3 interaction inhibited infiltration of lymphocytes into pancreatic islets and suppressed the onset of diabetes $[14,25]$. These data are from a virus-induced diabetes model in which CXCL10 is acutely produced after infection, and therefore the migration of possibly both effector as well as regulatory $\mathrm{T}$ cells has been blocked. Both effector and regulatory $\mathrm{T}$ cells might be attracted in excess, leading to disease acceleration, despite the possible presence of a higher absolute number of 'hybrid Tregs'. Hybrid Tregs producing CXCR3 might be more important in a spontaneous model with slow disease progression.

One might argue that the function of Tregs might be different between $\mathrm{Cxcr}^{-/-}$and wild-type NOD mice. However, Tgf- $\beta$, Ill10, Il4, Ifn- $\gamma$, Il-2 and Gzmb expression levels of sorted $\mathrm{FOXP}^{+}$cells (data not shown), as well as the suppressive function of $\mathrm{CD} 4^{+} \mathrm{CD} 25^{+}$cells in vitro, were essentially the same in the two strains. Therefore, we consider that the function of Tregs must be the same in the two strains.

Considering the clinical situation, after sorting this $\mathrm{CXCR}^{+}{ }^{+}$Treg population at the onset of diabetes, and the expansion of this population, these cells might be used to regulate the active disease state through transfer back into the donor. We examined whether Tregs from wild-type mice, which were considered to include $\mathrm{CXCR}^{+}$Tregs, could suppress diabetes development compared with Tregs from $\mathrm{CxCr}^{-/-}$mice (all CXCR3 ${ }^{-}$Tregs), using a cyclophosphamide-induced diabetes model to create the same condition in the recipients, and found that Tregs from wild-type mice suppressed the onset of diabetes in comparison with Tregs from $\mathrm{Cxcr} 3^{-/-}$mice. Therefore, $\mathrm{CXCR}^{+}$ Tregs (hybrid Tregs) seem to work in vivo to control diabetes development. We believe hybrid Tregs to be a very promising candidate cell population for the control of type 1 diabetes.

Funding This study was partly funded by a Grant-in-Aid for Scientific Research.

Duality of interest The authors declare that there is no duality of interest associated with this manuscript.

Author contributions All authors contributed to the conception and design, or analysis and interpretation of data, drafting the article or revised it critically, and approved the final version of the paper for publication. YY and YO (Y. Okubo) contributed to the experimental work. YY, YO (Y. Okubo) and AS drafted the article and revised it most critically.

\section{References}

1. Atkinson MA, Maclaren NK (1994) The pathogenesis of insulindependent diabetes mellitus. N Engl J Med 331:1428-1436

2. Shimada A, Rohane P, Fathman CG, Charlton B (1996) Pathogenic and protective roles of $\mathrm{CD} 45 \mathrm{RB}$ (low) $\mathrm{CD} 4+$ cells correlate with cytokine profiles in the spontaneously autoimmune diabetic mouse. Diabetes 45:71-78

3. Shimada A, Morimoto J, Kodama K et al (2001) Elevated serum IP-10 levels observed in type 1 diabetes. Diabetes Care 24:510-515

4. Shigihara T, Shimada A, Oikawa Y et al (2005) CXCL10 DNA vaccination prevents spontaneous diabetes through enhanced beta cell proliferation in NOD mice. J Immunol 175:8401-8408

5. Morimoto J, Yoneyama H, Shimada A et al (2004) CXC chemokine ligand 10 neutralization suppresses the occurrence of diabetes in non obese diabetic mice through enhanced beta cell proliferation without affecting insulitis. J Immunol 173:7017-7024

6. Lacotte S, Brun S, Muller S, Dumortier H (2009) CXCR3, inflammation, and autoimmune diseases. Ann N Y Acad Sci 1173:310-317

7. Rotondi M, Chiovato L, Romagnani S, Serio M, Romagnani R (2007) Role of chemokines in endocrine autoimmune diseases. Endocrine Rev 28:492-520 
8. Christensen JE, de Lemos C, Moos T, Christensen JP, Thomsen AR (2006) CXCL10 is the key ligand for CXCR3 on CD8+ effector $\mathrm{T}$ cells involved in immune surveillance of the lymphocytic choriomeningitis virus-infected central nervous system. J Immunol 176:4235-4243

9. Nicholas MW, Dooley MA, Hogan SL et al (2008) A novel subset of memory B cells is enriched in autoreactivity and correlates with adverse outcomes in SLE. Clin Immunol 126:189-201

10. Wilk E, Kalippke K, Buyny S, Schmidt RE, Jacobs R (2008) New aspects of NK cell subset identification and inference of NK cells' regulatory capacity by assessing functional and genomic profiles. Immunobiology 213:271-283

11. Müller M, Carter SL, Hofer MJ et al (2007) CXCR3 signaling reduces the severity of experimental autoimmune encephalomyelitis by controlling the parenchymal distribution of effector and regulatory $\mathrm{T}$ cells in the central nervous system. J Immunol 179:2774-2786

12. Frigerio S, Junt T, Lu B et al (2002) Beta cells are responsible for CXCR3-mediated $\mathrm{T}$ cell infiltration in insulitis. Nat Med 8:1414-1420

13. Christen U, McGavern DB, Luster AD, von Herrath NG, Oldstone MB (2003) Among CXCR3 chemokines, IFN-gamma-inducible protein of $10 \mathrm{kDa}(\mathrm{CXC}$ chemokine ligand (CXCL) 10) but not monokine induced by IFN-gamma (CXCL9) imprints a pattern for the subsequent development of autoimmune disease. J Immunol 171:6838-6845

14. Rhode A, Pauza ME, Barral AM et al (2005) Islet-specific expression of CXCL10 causes spontaneous islet infiltration and accelerates diabetes development. J Immunol 175:3516-3524

15. Koch MA, Tucker-Heard G, Perdue NR, Killebrew JR, Urdahl KB, Campbell DJ (2009) The transcription factor T-bet controls regulatory $\mathrm{T}$ cell homeostasis and function during type 1 inflammation. Nat Immunol 10:595-602

16. Hancock WW, Lu B, Gao W et al (2000) Requirement of the chemokine receptor CXCR3 for acute allograft rejection. J Exp Med 192:1515-1520

17. Yamamoto T, Yamato E, Tashiro F et al (2004) Development of autoimmune diabetes in glutamic acid decarboxylase 65 (GAD65) knockout NOD mice. Diabetologia 47:221-224

18. Turner MS, Kane LP, Morel PA (2009) Dominant role of antigen dose in $\mathrm{CD}^{+}{ }^{+} \mathrm{Foxp}^{+}$regulatory $\mathrm{T}$ cell induction and expansion. $\mathrm{J}$ Immunol 183:4895-4903

19. Rabinovitch A (1994) Immunoregulatory and cytokine imbalances in the pathogenesis of IDDM. Therapeutic intervention by immunostimulation? Diabetes 43:613-621

20. Katz JD, Benoist C, Mathis D (1995) T helper cell subsets in insulin-dependent diabetes. Science 268:1185-1188

21. Toyoda H, Formby B (1998) Contribution of T cells to the development of autoimmune diabetes in the NOD mouse model. Bioessays 20:750-757

22. Delovitch TL, Singh B (1997) The nonobese diabetic mouse as a model of autoimmune diabetes: immune dysregulation gets the NOD. Immunity 7:727-738

23. Gao JM, Lu B, Guo ZJ (2006) CXC chemokine receptor 3 modulates bleomycin-induced pulmonary injury via involving inflammatory process. Chin Med Sci J 21:152-156

24. Yamada S, Irie J, Shimada A et al (2003) Assessment of beta cell mass and oxidative peritoneal exudate cells in murine type 1 diabetes using adoptive transfer system. Autoimmunity $36: 63-70$

25. Christen U, Benke D, Wolfe T et al (2004) Cure of prediabetic mice by viral infections involves lymphocyte recruitment along an IP-10 gradient. J Clin Invest 113:74-84 